\title{
Photoexcited states in correlated band insulators
}

\author{
Nagamalleswararao Dasari ${ }^{1,2, *}$ and Martin Eckstein ${ }^{1}$ \\ ${ }^{1}$ Department of Physics, University of Erlangen-Nuremberg, 91058 Erlangen, Germany \\ ${ }^{2}$ Max Planck Institute for the Structure and Dynamics of Matter, 22761 Hamburg, Germany
}

(Received 16 February 2018; revised manuscript received 21 June 2018; published 11 July 2018)

\begin{abstract}
We study the photoexcitation dynamics of correlated band insulators, using nonequilibrium dynamical meanfield theory for the ionic Hubbard model. We find two distinct behaviors, depending on the ratio of the on-site interaction $U$ and the bare band gap $\Delta$. For small interactions, the relaxation is characterized by intraband carrier scattering in relatively rigid bands, leading to a nonthermal intermediate state with separate thermal distributions of electrons and holes. This behavior can be viewed as typical for a band insulator with weak interactions. For larger interaction, on the other hand, we observe a strong modification of the electronic spectrum and a filling-in of the gap after photoexcitation, along with a rapid thermalization of the system. The two behaviors therefore provide a dynamical distinction of a correlated band insulator and a band insulator, which can differ even when the spectra of the two systems are similar in equilibrium. The crossover happens when the interaction $U$ is comparable to $\Delta$.
\end{abstract}

DOI: 10.1103/PhysRevB.98.035113

\section{INTRODUCTION}

The possibility to switch quantum states through ultrafast nonthermal pathways has made nonequilibrium studies of quantum many-body systems attractive to the condensedmatter community [1]. Using laser photoexcitation or external electric fields, one can induce transient states on short timescales and, in some cases, even long-lived hidden quantum states with electronic properties which are entirely different from equilibrium [2-5].

A rich variety of novel phases in nonequilibrium can be expected for strongly correlated systems. Electronic correlations are predominantly studied in systems which are metallic in the noninteracting limit. In these systems, correlations result in phenomena like the Mott transition, high-temperature superconductivity, and non-Fermi-liquid behavior. In band insulators, in contrast, one may naively expect that the existence of the band gap and the absence of low-energy quasiparticles reduces the importance of electronic correlations. However, this turns out to be not true. The role of electronic correlations in band insulators has been studied in various models, including the ionic Hubbard model [6-20], a two-orbital Hubbard model with crystal-field splitting [21], a two-sublattice model with interorbital hybridization [22,23], and a bilayer model with two identical Hubbard planes [24-28]. In general, at weak coupling the competition between the local Coulomb interaction and the noninteracting band gap results in a correlated band insulator with a renormalized gap. Further increasing the interaction strength can even close the gap and lead to an interaction-driven metal or bond-ordered state in the strongcoupling limit $[13,14,22]$. The renormalized band gaps and the difference in charge and spin gaps distinguishes correlated band insulators from noninteracting band insulators $[9,10,22]$.

*nagamalleswararao.d@gmail.com
Correlated band insulators provide a paradigm example for systems with competing ground-state interactions and hence are good candidates to explore hidden quantum states through nonthermal pathways.

In a band insulator, photoexcitation will promptly lead to a partial occupation of the conduction band. At weak coupling, one may expect the dynamics of such systems to be similar to semiconductors: Interactions provide a mechanism for electron-electron scattering, while the bands are almost rigid apart from some photoinduced screening of the gap which is largely included in a Hartree shift of the bands. This leads to a state in which electrons and holes are separately thermalized in the conduction and valence bands. On longer timescales, the system would establish a common temperature and chemical potential, and the energy would be passed to the lattice. Such processes in semiconductors are well described using quantum Boltzmann equations [29]. In this paper, we explore how this picture is modified at larger interaction and whether there is a distinct dynamical behavior of band insulators and correlated band insulators. How is the gap renormalized or even filled due to local correlations after photoexcitations? What is the role of these correlations in the thermalization process: Does the filling in of the gap lead to a speedup of thermalization, and is there room for nonthermal metallic states?

The theoretical study of such systems out of equilibrium is challenging. The dynamics of interacting quantum systems often can be described using quantum kinetic equations [29]. However, this approach assumes the existence of well-defined quasiparticles which change their distribution in a rigid band due to collisions. The rigid-band assumption can break down in correlated systems because the local Coulomb interactions can strongly renormalize the electronic spectrum. The quantum kinetic equation approach fails to describe a change in the electronic band structure due to local correlations, while it can describe the relaxation towards a thermal state on the long timescale [30]. In the past few years, nonequilibrium 
dynamical mean-field theory (DMFT) has been widely applied to study the photoexcitation and quench dynamics of correlated electronic systems [31]. In this work, we study the dynamics of correlated band insulators by means of nonequilibrium DMFT, using iterative perturbation theory (IPT) as an impurity solver. We find a crossover between two different behaviors when the local Coulomb interaction becomes comparable to the noninteracting band gap. The typical behavior of a band insulator with two separate subsystems of thermalized holes and electrons persists only for the smaller interaction.

This paper is organized as follows. In Sec. II, we present the model and method used to study the photoexcitation of correlated band insulators and briefly discuss the equilibrium properties of the model. In Sec. III we present the numerical results, and Sec. IV contains an analysis and discussion.

\section{MODEL AND METHOD}

\section{A. Ionic Hubbard model and DMFT}

Correlated band insulators are well described by the ionic Hubbard model (IHM) on a bipartite lattice with two sublattices, $A$ and $B$. The Hamiltonian is given by

$$
\begin{aligned}
\mathcal{H}= & -J(t) \sum_{\langle i, j\rangle, \sigma}\left[c_{i, \sigma}^{\dagger} c_{j, \sigma}+\text { H.c. }\right]+\Delta \sum_{i \in A} n_{i}-\Delta \sum_{i \in B} n_{i} \\
& +U \sum_{i} n_{i \uparrow} n_{i \downarrow}-\mu \sum_{i} n_{i},
\end{aligned}
$$

where $J$ is the nearest-neighbor hopping amplitude and $U$ is the on-site Coulomb interaction. The staggered ionic potential $\Delta$ is the origin of the noninteracting band gap. We choose the chemical potential $\mu$ such that total filling of the system is fixed at half filling $\left(\sum_{\sigma}\left[n_{A, \sigma}+n_{B, \sigma}\right]=2\right)$. At half filling, the above Hamiltonian describes a band insulator and a Mott insulator in the limits $(U=0, \Delta \neq 0)$ and $(U=\infty, \Delta=0)$, respectively. Nontrivial states arise when both $\Delta$ and $U$ are nonzero and finite.

To study the photoexcitation dynamics of correlated band insulators we employ the nonequilibrium DMFT formalism [31], which is exact in the limit of the infinite-coordination number $Z$ with hopping $J(t)=J^{*} / \sqrt{Z}$. We choose a noninteracting dispersion of bands on a bipartite lattice, with a semielliptical density of states $\rho(\epsilon)=\frac{1}{\pi J^{*}} \sqrt{4 J^{* 2}-\epsilon^{2}}$. We set the half bandwidth $2 J^{*}=1$ as an energy unit, and the unit of time is $\frac{\hbar}{2 J^{*}}$. The DMFT formalism makes a local approximation for the self-energy, i.e., $\Sigma_{i j}\left(t, t^{\prime}\right) \approx \Sigma_{i i}\left(t, t^{\prime}\right)$, which allows us to map the lattice problem (1) to an effective quantum impurity problem with a self-consistently determined bath $\Gamma\left(t, t^{\prime}\right)$. The impurity action for sublattice $\alpha \in\{A, B\}$ is given by

$$
S_{\alpha}=\int_{C} d t H_{\mathrm{loc}}^{\alpha}(t)+\sum_{\sigma} \int_{C} d t d t^{\prime} c_{\sigma \alpha}^{\dagger}(t) \Gamma_{\alpha}\left(t, t^{\prime}\right) c_{\sigma \alpha}(t) .
$$

Here $C$ is the Keldysh time contour (for an introduction to the Keldysh formalism, see, e.g., Ref. [31]), and

$$
H_{\mathrm{loc}}^{\alpha}=U n_{\uparrow}^{\alpha} n_{\downarrow}^{\alpha}+\left[\Delta_{\alpha}-\mu\right]\left(n_{\uparrow}^{\alpha}+n_{\downarrow}^{\alpha}\right)
$$

is the local Hamiltonian on the impurity, with $\Delta_{A}=\Delta$ and $\Delta_{B}=-\Delta$. The semielliptical density of states yields a closed-form expression for the self-consistency relation, which is given by $\Gamma_{\alpha}\left(t, t^{\prime}\right)=J^{*}(t) G_{\bar{\alpha}}\left(t, t^{\prime}\right) J^{*}\left(t^{\prime}\right)$, where $G_{\alpha}\left(t, t^{\prime}\right)=$ $-i\left\langle\mathrm{~T}_{C} c_{\alpha}(t) c_{\alpha}^{\dagger}\left(t^{\prime}\right)\right\rangle_{S_{\alpha}}$ is the local Green's function defined on the Keldysh contour ( $\bar{\alpha}=B$ for $\alpha=A$ and vice versa). For a given sublattice hybridization $\Gamma_{\alpha}$, the local Green's functions $G_{\alpha}$ can be determined from the Dyson equation,

$$
\left[i \partial_{t}+\mu+\Delta_{\alpha}-\Sigma_{\alpha}-\Gamma_{\alpha}\right] G_{\alpha}=1,
$$

where $\Sigma_{\alpha}$ is the local self-energy which is calculated from the effective impurity problem. We use the real-time iterative perturbation theory as an impurity solver to calculate the local self-energy. The diagrammatic expression for the IPT ansatz is given by

$$
\begin{aligned}
\Sigma_{\alpha}\left(t, t^{\prime}\right)= & \sqrt{A^{\alpha}(t) A^{\alpha}\left(t^{\prime}\right)} U(t) U\left(t^{\prime}\right) \\
& \times \mathcal{G}_{\alpha}^{H}\left(t, t^{\prime}\right) \mathcal{G}_{\alpha}^{H}\left(t, t^{\prime}\right) \mathcal{G}_{\alpha}^{H}\left(t^{\prime}, t\right),
\end{aligned}
$$

where $\mathcal{G}_{\alpha}^{H}$ is the Hartree-corrected bath propagator, which is given by the Dyson equation

$$
\left[i \partial_{t}+\mu-\Delta_{\alpha}-\Sigma_{H}^{\alpha}-\Gamma_{\alpha}\right] \mathcal{G}_{\alpha}^{H}=1,
$$

with the Hartree self-energy $\Sigma_{H}^{\alpha}\left(t, t^{\prime}\right)=U n^{\alpha}(t) \delta_{c}\left(t, t^{\prime}\right)$. Following Ref. [13], the factors $A^{\alpha}$ in Eq. (5) are chosen such that in equilibrium the ansatz is exact in the weak-coupling limit $(U / t \ll 1)$ and it has the exact short-time behavior for all values of $U / t$, which imposes various exact sum rules. The corresponding expression for $A^{\alpha}(t)$ is $n_{\alpha}(t)\left[1-n_{\alpha}(t)\right] / n_{\alpha}^{0}(t)[1-$ $\left.n_{\alpha}^{0}(t)\right]$, where $n_{\alpha}^{0}(t)$ is the impurity occupancy, which is calculated from $\mathcal{G}_{\alpha}^{H}$. Writing the $A$-factor in the symmetrized form $\sqrt{A^{\alpha}(t) A^{\alpha}\left(t^{\prime}\right)}$ guarantees that the self-energy equation (5) is Hermitian, while the expression reduces to the conventional form $\sqrt{A^{\alpha}(t) A^{\alpha}\left(t^{\prime}\right)}=A_{\alpha}$ at equilibrium, when $A_{\alpha}$ is time independent.

Nonequilibrium DMFT measures the local Green's function for each sublattice. The sublattice occupancy is obtained from the equal-time lesser component of the Green's function, $m(t)=n_{A}(t)-n_{B}(t)=-i G_{A}^{<}(t, t)+i G_{B}^{<}(t, t)$. To find the effective temperature of the photoexcited system we need to calculate the total energy of the system, which is the sum of kinetic energy, interaction energy, and lattice potential energy. The kinetic energy of a photoexcited system is given by the equal-time contour convolution of the hybridization function with the local Green's function, $E_{\mathrm{kin}}=-i \sum_{\alpha}\left[\Gamma_{\alpha} *\right.$ $\left.G_{\alpha}\right]^{<}(t, t)$, and the interaction energy is the equal-time contour convolution of the local self-energy with the local Green's function, $E_{\text {int }}=i \sum_{\alpha}\left[\Sigma_{\alpha} * G_{\alpha}\right]^{<}(t, t)$ [31]. The lattice potential energy is $E_{\Delta}(t)=\Delta\left[n_{A}(t)-n_{B}(t)\right]$. The double occupancy $d_{\alpha}(t)=\left\langle n_{\uparrow}^{\alpha} n_{\downarrow}^{\alpha}\right\rangle$ is obtained from the interaction energy.

One of the issues with IPT is the nonconserving nature of the self-energy in the sense of Baym-Kadanoff. A violation of the energy conservation can be identified when there is a drift of the total energy with time even though the Hamiltonian is time independent. In previous nonequilibrium DMFT calculations for interaction quenches in a single-band Hubbard model it was found that IPT conserves the total energy very accurately up to all accessible simulation times in the weak-to-intermediatecoupling regime, while with increasing $U$ there is a relatively sharp crossover after which only the short-time dynamics is correctly captured [32]. In this work we focus on relatively weak coupling, and we have confirmed that an unphysical 
short-time increase of the energy as observed in Ref. [32] does not occur.

\section{B. Equilibrium DMFT study of the IHM}

A first understanding of the equilibrium states of the IHM can be obtained from the atomic limit $J / U=0$ at total filling equal to 1 . In this trivially soluble limit, the ground state for $U<2 \Delta$ has two electrons on sublattice $B$ and zero on sublattice $A$, resulting in a band insulator with a band gap $\Delta-U / 2$. In the opposite limit $(U>2 \Delta)$ each sublattice is occupied by one electron, and we get a Mott insulator with a gap $U$. In the atomic limit, the system is gapless at $U=2 \Delta$. An interesting question therefore is whether local correlations broaden this metallic point into a metallic phase when $J$ is nonzero.

Equilibrium studies of the IHM at zero temperature using DMFT with an IPT impurity solver find that electronic correlations strongly renormalize the noninteracting band gap for small values of $U$. The gap in the spectral function vanishes at a critical interaction $U_{c}$, and the system is metallic for a finite range of interactions $[13,15]$. As we further increase $U>U_{c}$, the system becomes Mott insulating; that is, the gap in the spectral function opens again and increases with $U$. The crossover from a correlated band insulator to a metal has been observed experimentally in photoemission spectra of $\mathrm{SrRu}_{1-x} \mathrm{Ti}_{x} \mathrm{O}_{3}$ [33].

IPT studies of the IHM find the metallic phase only up to intermediate values of $\Delta$, while for large values of the ionic potential there is a direct transition from a band insulator to a Mott insulator $[13,15]$. A recent study of the IHM at finite temperature using a continuous-time quantum Monte Carlo (CTQMC) impurity solver obtained a phase diagram similar to IPT for intermediate values of $\Delta$, but in contrast to IPT the intermediate metallic phase persists to even larger values of $\Delta$ [18]. An open issue is the nature of the intermediate metallic phase [15]. Two-dimensional cluster extensions of DMFT find a bond-ordered phase as an intermediate metallic phase [14], while single-site DMFT using CTQMC suggests that the intermediate metallic phase is a Fermi liquid [18].

\section{Photodoping}

Photodoping changes the electron-hole concentration by irradiating light on the sample. Experimentally, laser photoexcitation not only changes the carrier concentration of the bands at the Fermi level but can also excite electrons from bands far below the Fermi level into the conduction band and electrons from the valence band to other bands far above the Fermi level. In our work, we employ this setup to induce the photoexcited state. To mimic such an experimental situation theoretically, one can couple two additional wideband particle reservoirs to the system. One reservoir is entirely filled and will therefore inject particles into the empty states of the system, while the other one is empty and can take out electrons from occupied states. The additional fermionic baths are noninteracting and can be integrated out exactly. The resulting DMFT action is the same as Eq. (2), but the hybridization function is modified to

$$
\Gamma_{\alpha}^{\prime}\left(t, t^{\prime}\right)=\Gamma_{\alpha}\left(t, t^{\prime}\right)+\Gamma^{\text {filled }}\left(t, t^{\prime}\right)+\Gamma^{\text {empty }}\left(t, t^{\prime}\right) .
$$

Here the first term corresponds to the self-consistent hybridization of the ionic Hubbard model, and the last two terms correspond to the hybridization of the filled and empty reservoirs. The latter are given by

$$
\Gamma^{\text {filled/empty }}\left(t, t^{\prime}\right)=h(t) G^{\text {filled/empty }}\left(t, t^{\prime}\right) h\left(t^{\prime}\right),
$$

where $G^{\text {filled/empty }}$ is the Green's function of the reservoir and $h(t)$ is the time profile of the coupling between the IHM and the reservoirs, for which we take the form

$$
h(t)=h_{0} \sin \left(\pi t / t_{\text {pulse }}\right)
$$

for $t \leqslant t_{\text {pulse }}$ and $h(t)=0$ for $t>t_{\text {pulse }}$. Throughout this work we fix $t_{\text {pulse }}=3.0$, and $h_{0}$ is chosen to control the excitation density. The density of states corresponding to $G^{\text {filled/empty }}\left(t, t^{\prime}\right)$ is a Lorentzian of width $8 J^{*}$.

\section{RESULTS}

We start the analysis of relaxation after photoexcitation with an investigation of the time-dependent double occupancy and the sublattice occupation (Secs. III A and III B) and then proceed to analyze spectral functions and the occupation function (Sec. III C). To know whether the photoexcited system thermalizes after the pulse, we need to find the effective temperature $T_{\text {eff }}$ of the time-evolved state [34]. For this purpose, we determine a thermal equilibrium state which has the same energy as the time-evolved state. The corresponding temperature of the equilibrium state then defines $T_{\text {eff. }}$. (In practice, we choose the excitation density in order to fix a given effective temperature, $T_{\text {eff }}=1$ or $T_{\text {eff }}=0.3$.) With this we can compare time-dependent expectation values of various observables $O(t)$ to corresponding expectation values $O_{\text {th }} \equiv$ $O\left(T_{\text {eff }}\right)=\operatorname{Tr}\left[\exp ^{-H / T_{\text {eff }}} O\right] / Z$. Throughout the paper we fix a low initial temperature $T$ by $\beta=1 / T=32$.

\section{A. Thermalization of the metal $(\Delta=0)$}

When the ionic potential is zero, the IHM reduces to a simple one-band Hubbard model with intraorbital interaction $U$, which is metallic at small values of $U$. In order to later contrast the results to the correlated band insulator, we first investigate the thermalization of this metallic phase within our formalism. In Fig. 1(a), we plot the time-dependent double occupancy $d(t)=\left\langle n_{\uparrow} n_{\downarrow}\right\rangle$ of the photoexcited system for different values of $U$. We fit the time-dependent double occupancy to a single exponential function $d(t)=d_{t=\infty}+b \exp \left(-t / \tau_{d}\right)$ and extract the extrapolated value $d_{t=\infty}$ of $d(t)$ in the long-time limit and the corresponding relaxation times $\tau_{d}$. The thermal values $d_{\text {th }}$ of the double occupancy, obtained from equilibrium simulations at the corresponding effective temperature, are shown by arrows in Fig. 1(a). The difference $\left|d_{\mathrm{th}}-d_{t=\infty}\right|$ is plotted in Fig. 1(b). One can see that the time-dependent double occupancy approaches $d_{\text {th }}$ in the long-time limit for all values of $U$ within our numerical accuracy, which implies thermalization of the weakly correlated metal. The relaxation times $\tau_{d}$ are plotted in Fig. 1(c). The thermalization times can be fit well with a power law $\tau_{d} \sim 1 / U^{2}$. This behavior is consistent with a quasiparticle picture: In the different calculations we have chosen the excitation such that the effective temperature is fixed to $T_{\text {eff }}=1$, so that the phase space for scattering is fixed, 


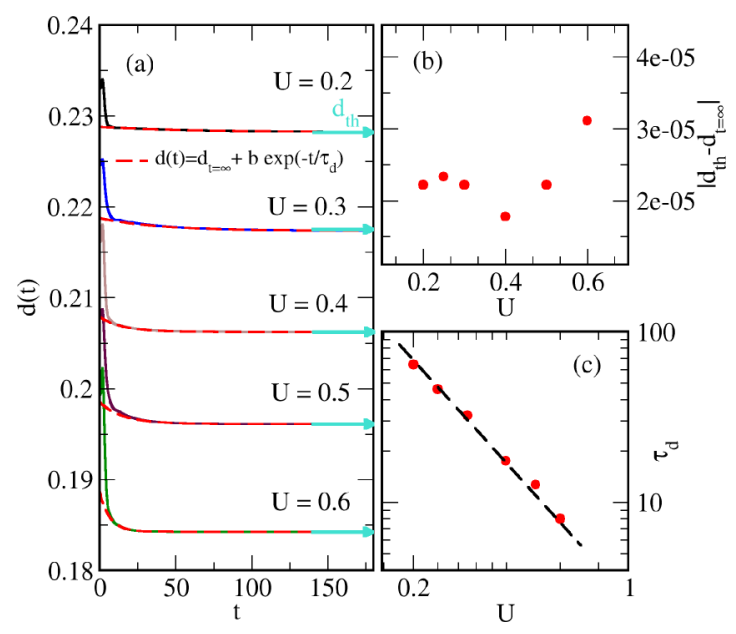

FIG. 1. (a) Double occupancy of the photoexcited system for different values of $U$. The excitation density is chosen such that $T_{\text {eff }}=1$. Solid curves show the numerical data, and red dashed lines show exponential fits. Small arrows indicate the double occupancy in thermal equilibrium. Zero time $(t=0)$ is the starting time of simulations. (b) Difference of the double occupancy in equilibrium at temperature $T=T_{\text {eff }}$ and in the photoexcited system. (c) Relaxation time of the double occupancy for different values of $U$. The dashed curve is a fit to the relaxation times with the relation $\frac{a}{U^{2}}$.

and the scattering rate is given by the scattering matrix element, which is proportional to $U^{2}$.

\section{B. Correlated band insulator: Relaxation of the double occupancy and the sublattice occupation}

To study the relaxation dynamics of the correlated band insulator, we analyze the relaxation dynamics of the timedependent double occupancy $d^{A}(t)$ (measured at the $A$ sublattice without loss of generality). Like in the previous section, we extract the value of the double occupancy at infinite time
$d_{t=\infty}^{A}$ and the relaxation time $\tau_{d}^{A}$ from an exponential fit $d^{A}(t)=d_{t=\infty}^{A}+b \exp \left(-t / \tau_{d}^{A}\right)$. In the top panels of Fig. 2, we plot the difference of $d_{\mathrm{th}}^{A}$ from the time-evolved value $d_{t=\infty}^{A}$ for different values of the ionic potential. In contrast to the metal, $\left|d_{\mathrm{th}}^{A}-d_{t=\infty}^{A}\right|$ approaches zero only when $U / \Delta \gtrsim 1$. This implies that for smaller interactions, the observed relaxation on the timescale of our simulation is towards a nonthermal state, while the behavior at large interactions is consistent with thermalization. The crossover from nonthermal to thermal behavior occurs roughly when $U$ is of the order of $\Delta$, but it also depends on the excitation density (i.e., the effective temperature of the final state). In the bottom panels of Fig. 2, we plot the relaxation times $\tau_{d}^{A}$ obtained from the exponential fits. In the regime where the system thermalizes $(U / \Delta \gtrsim 1)$, the relaxation times decrease with $U$, like in the metal. In the opposite limit, the relaxation depends on $U$ in a nonmonotonous way and reaches a maximum value around $U / \Delta \approx 0.6$ for $\Delta=0.4$. As we increase $\Delta$, the maximum in the relaxation time shifts gradually towards higher values of $U / \Delta$. As observed for $\left|d_{\mathrm{th}}^{A}-d_{t=\infty}^{A}\right|$, this maximum also depends on the excitation density. The maximum in the relaxation time corresponds to a value of $U$ where the intraband relaxation within the bands and the relaxation towards a thermal state happens at similar timescales. Therefore a fit at the single exponential becomes less reliable, which makes a quantitative discussion of the crossover between the thermal and nonthermal regimes difficult.

The sublattice occupancy $m(t)=n_{A}(t)-n_{B}(t)$ exhibits a behavior similar to that observed for the double occupancy. For this purpose, we again analyze $m(t)$ by means of an exponential fit, $m(t)=m_{t=\infty}+b \exp \left(-t / \tau_{m}\right)$. The top panels of Fig. 3 show the difference between $m_{\text {th }}$ and $m_{t=\infty}$ for the same parameters as in Fig. 2. Similar to the double occupancy, the sublattice occupation $m(t)$ displays a crossover from nonthermal to thermal behavior when $U$ is comparable to the ionic potential, with the same trend in the relaxation times $\tau_{m}$ (bottom panel of Fig. 3).
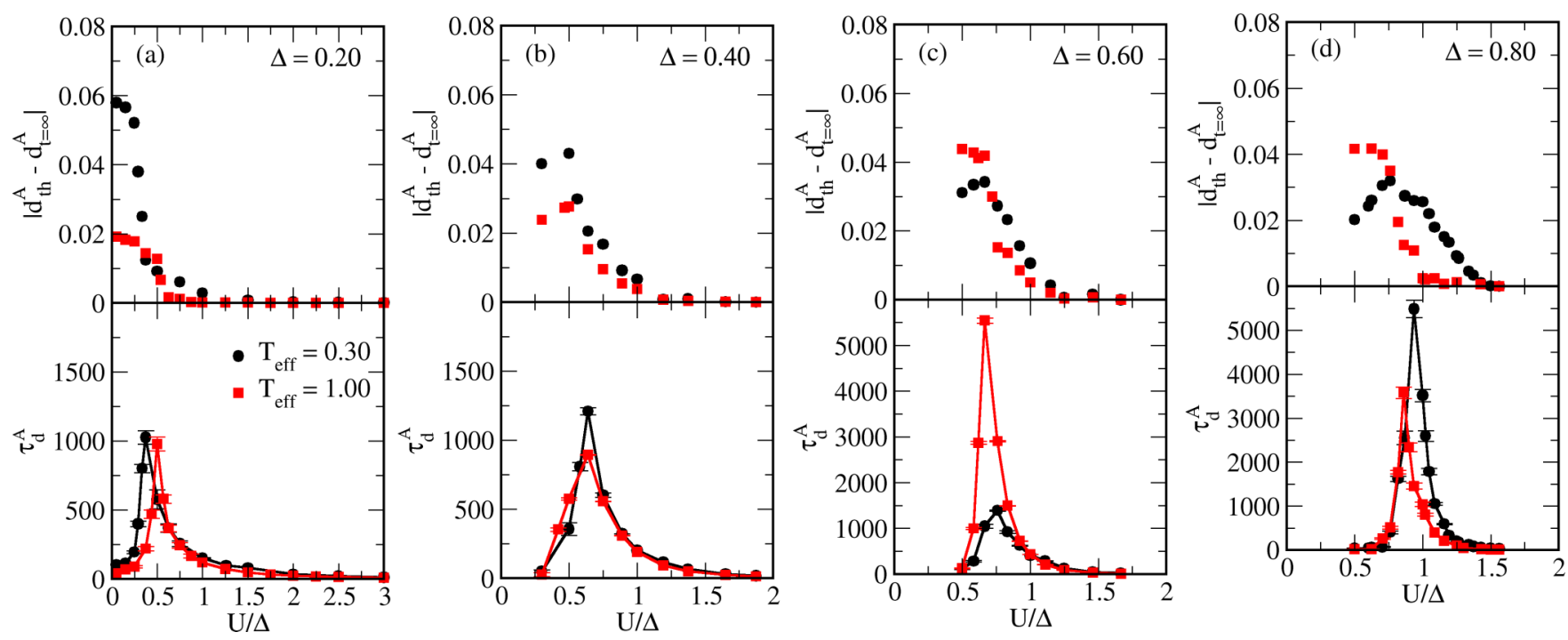

FIG. 2. Top: Difference of the double occupancy in thermal equilibrium at temperature $T=T_{\text {eff }}$ and in the time-evolved state for different values of the ionic potential: (a) $\Delta=0.2$, (b) $\Delta=0.4$, (c) $\Delta=0.6$, and (d) $\Delta=0.8$. The excitation density is chosen such that $T_{\text {eff }}=0.3$ (black circles) or $T_{\text {eff }}=1.0$ (red squares). Bottom: Relaxation times of the double occupancy. 

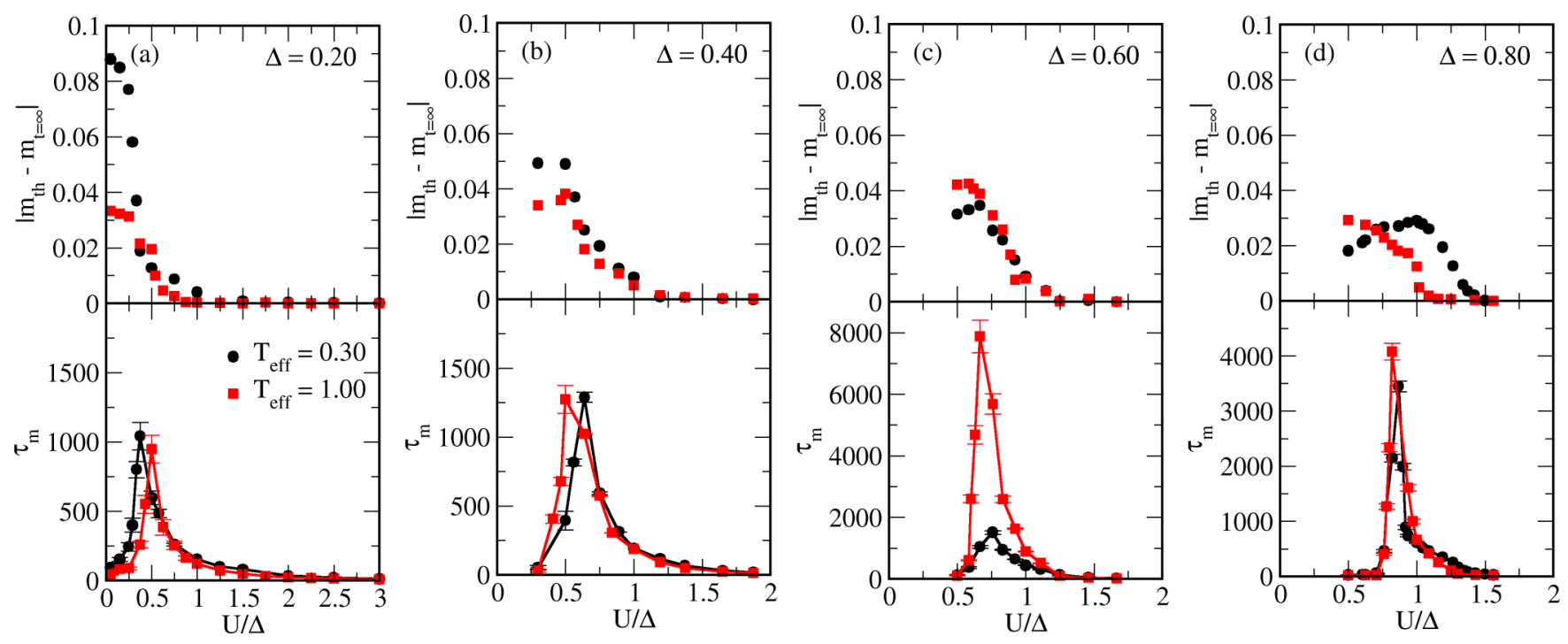

FIG. 3. Top: Difference of the sublattice occupancy in thermal equilibrium $\left(m_{\mathrm{th}}\right)$ at temperature $T=T_{\text {eff }}$ and in the time-evolved state $\left(m_{t=\infty}\right)$ for different values of the ionic potential: (a) $\Delta=0.2$, (b) $\Delta=0.4$, (c) $\Delta=0.6$, and (d) $\Delta=0.8$. The excitation density is chosen such that $T_{\text {eff }}=0.3$ (black circles) or $T_{\text {eff }}=1.0$ (red squares). Bottom: Relaxation times of the sublattice occupancy.

\section{Relaxation of the spectral function}

The observed crossover from nonthermal to thermal behavior can be further analyzed by looking at the spectral functions and the occupation function. The analysis of the spectral function furthermore allows us to access the possible transient metallicity of the photodoped state. To identify any such metallic states we calculate the single-particle spectral function $A^{R}(\omega, t)$ and the occupied density of states $A^{<}(\omega, t)$ from a Fourier transform,

$$
A^{\gamma}(t, \omega)=\mp \frac{1}{\pi} \operatorname{Im} \int_{0}^{t} d s e^{i \omega s} G^{\gamma}(t, t-s) .
$$

Here the upper and lower signs correspond to the spectral function $(\gamma=R)$ and the occupied density of states $(\gamma=<)$, respectively. The occupied density of states is closely related to the intensity in time-dependent photoemission spectroscopy [35]. Note that in nonequilibrium, we can define the Fourier transform of a function $G\left(t, t^{\prime}\right)$ with respect to the time difference in forward $\left(t^{\prime}>t\right)$, backward $\left(t^{\prime}<t\right)$, or symmetric fashion. Since we analyze spectra at the largest time when the system is almost in a steady state, the differences are minor, and we have chosen the backward form (10) unless stated otherwise.

In the left panels of Figs. 4(a) and 4(b), we compare the time-dependent spectral function in the photodoped state (green solid lines) and in the equilibrium state (magenta dashed lines) for $\Delta=0.4$ [Fig. 4(a)] and $\Delta=0.6$ [Fig. 4(b)] and different values of $U$. The corresponding occupation functions are shown in the right panels. In the thermal spectra, we find a well-defined gap at small values of $U$. The gap is robust even though the temperature is larger than $U$ and $\Delta$; that is, the main effect of the temperature is the occupation of states in the upper band, as seen from the occupied density of states in the right panel. Increasing values of $U$ lead to a renormalization of the gap and a broadening of the square-root singularity at the gap edge. When $U$ is of the order of the ionic potential, the gap in the spectral function starts to fills, until it is completely melted for large $U$. Hubbard bands would emerge only at larger values of $U$. Like for the double occupancy, a significant difference between thermal and photoexcited systems in $A^{<}(\omega)$ is apparent only for $U \lesssim \Delta$, confirming the nonthermal nature of the photoexcited state in this regime. Interestingly, the difference between thermal and photoexcited states is almost invisible for the retarded spectral function on the energy scale plotted in the left panels of Figs. 4(a) and 4(b), which will be discussed below (Sec. IV A).

The thermalization can be further verified by checking whether the electronic Green's functions satisfy the fluctuation-dissipation theorem (FDT). In equilibrium, the FDT implies a ratio $A^{<}(\omega) / A^{>}(\omega)=e^{-\beta(\omega-\mu)}$ between the occupied density of states $A^{<}(\omega)=-i G^{<}(\omega)$ and the unoccupied density of states $A^{>}(\omega)=i G^{>}(\omega)$ or, equivalently, a ratio $A^{<}(\omega) / A^{R}(\omega)=1 /\left(1+e^{\beta(\omega-\mu)}\right)$ between the occupied density of states and the spectral function. To test whether the photodoped state is in a quasiequilibrium state, we therefore calculate the partial Fourier transform $A^{<,>}(t, \omega)$ as in Eq. (10) and evaluate the quantity

$$
F_{A}(t, \omega)=\ln \left(\frac{A^{<}(t, \omega)}{A^{>}(t, \omega)}\right),
$$

which reduces to $-(\omega-\mu) / T_{\text {eff }}$ in a quasiequilibrium state. In Fig. 5, we plot $F_{A}(t, \omega)$ for a given $\Delta$ and different values of $U$. The FDT is satisfied when the local Coulomb interaction is greater than the ionic potential $U>\Delta$, which confirms that the photoexcited electrons have reached an equilibrium state. Furthermore, in this limit the effective temperature obtained from the FDT analysis accurately matches the value $T_{\text {eff }}=1$ obtained from the total energy.

For $U \lesssim \Delta$, however, the electronic distribution functions are highly nonthermal. Nevertheless, $F_{A}(t, \omega)$ takes a linear form $(a+b \omega)$ in the spectral region outside the gap $(|\omega| \gtrsim$ $0.3)$. The linear form of $F_{A}(t, \omega)$ directly implies that the electronic distribution functions are Fermi-Dirac. In general, we can decompose the occupied and nonoccupied densities of 

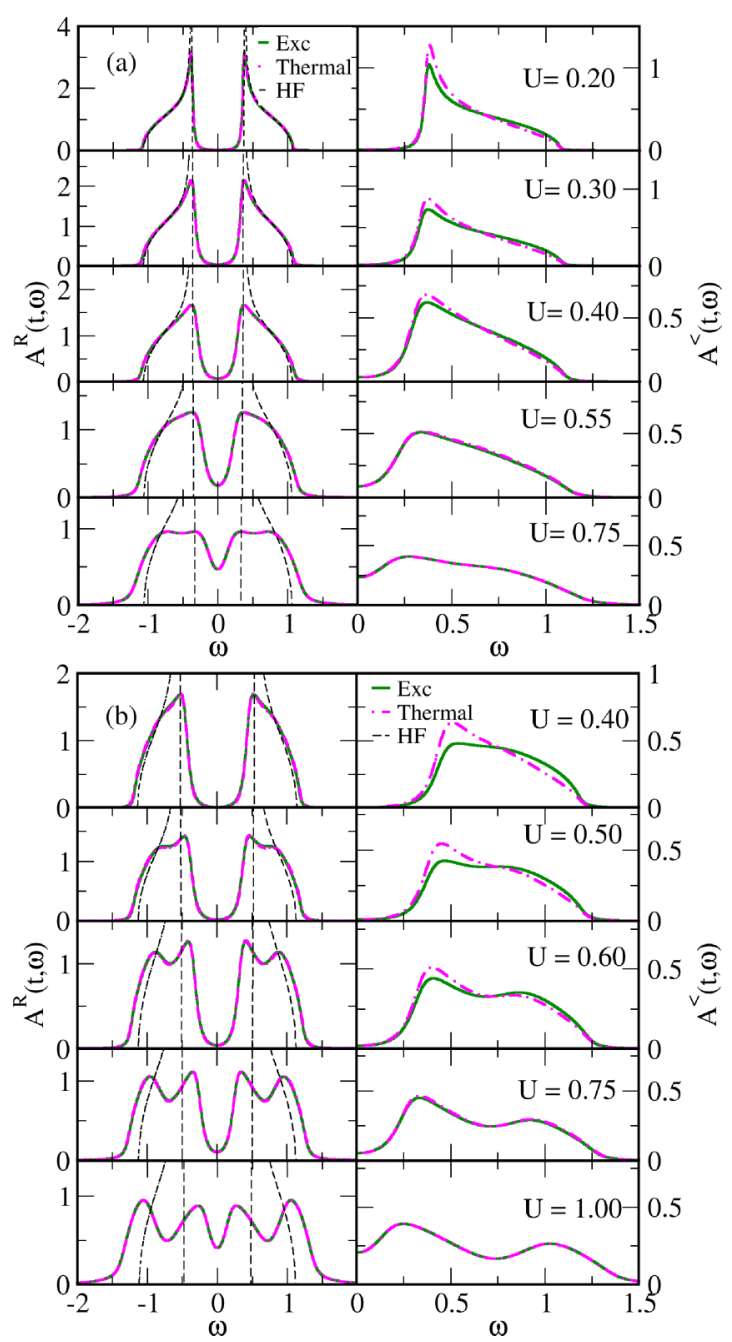

FIG. 4. (a) Left: Spectral function $A^{R}(t=180, \omega)$ for different values of $U$ and $\Delta=0.4$. Right: The corresponding occupied density of states $A^{<}(t=180, \omega)$ above the Fermi level. The green and pink curves correspond to the spectral functions of the excited system (Exc) and the system in thermal equilibrium (Thermal), respectively. The black dashed lines indicate the Hartree-Fock spectral function (see text). (b) Same as (a), but for $\Delta=0.6$. The excitation density is chosen such that $T_{\text {eff }}=1$.

states as $G_{A}^{<}=2 \pi i A(\omega) f(\omega)$ and $G_{A}^{>}=2 \pi i A(\omega)[1-f(\omega)]$ in terms of spectrum $A(\omega)$ and a distribution function $f(\omega)$. The relation $\ln \left(F_{A}\right)=\ln \left(G_{A}^{<} / G_{A}^{>}\right)=-(\omega-\mu) / T_{\text {eff }}$ thus directly implies $f(\omega)=\frac{1}{e^{(\omega-\mu) \beta_{\text {eff }}+1}}$. We find such a linear form for the function $F_{A}$ in the spectral region outside the gap for positive and negative frequencies. It can be interpreted in the form of two distinct Fermi functions with the same effective temperature $T_{\text {eff }}$ but with different chemical potentials in the upper and lower bands, respectively. This signals thermalization of electrons and holes as two separate subsystems due to intraband scattering. The effective temperatures of such photodoped states are very large (the distribution functions are almost flat) or even negative, corresponding to a population inversion. In photodoped Mott insulators with long-range Coulomb interactions, self-sustained population inversions have been found in the photodoped state [36]. In the present

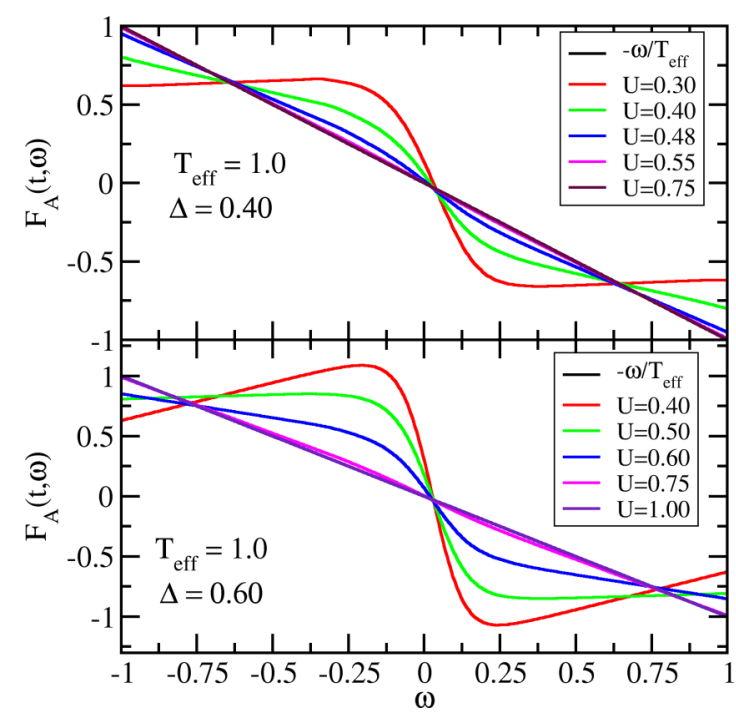

FIG. 5. Electronic distribution functions $F_{A}(t=180, \omega)$ [Eq. (11)] for different values of $U$ and ionic potential $\Delta=0.4$ (top panel) and $\Delta=0.6$ (bottom panel).

case, however, the effective temperature is mainly governed by the carrier distribution during the photodoping process.

\section{DISCUSSION}

\section{A. Crossover from a band insulator to a correlated band insulator}

Summarizing the previous section, we can say that the behavior of the system for $U \lesssim \Delta$ is well described by the expectation for a band insulator, which is characterized by the following features:

(i) At temperatures of interest for photoexcitation, the (retarded) spectral function is relatively rigid. Up to a trivial Hartree shift, which depends only on the thermal or photoinduced sublattice occupation, it is barely influenced by increasing the temperature or photodoping. This is seen by a comparison of thermal, photoexcited, and Hartree spectra in Fig. 4 . The fact that the spectra only weakly depend on the occupation also explains why the difference between thermal and photoexcited spectra is small in this regime.

(ii) The main relaxation mechanism is intraband relaxation, i.e., scattering between electrons and holes. Only a few scattering processes lead to a redistribution of carriers across the gap. This is consistent with the establishment of separate thermalized distributions in the region of the upper and lower bands, as shown in Fig. 5. The absence of a global thermal state with a common chemical potential also explains the nonthermal behavior observed in the double occupancy and the sublattice occupation, which are quantities that involve both valence- and conduction-band states and thus can indicate global thermalization.

Above the crossover $U \approx \Delta$, the behavior of the system changes drastically. In this regime, photoexcitation leads to a partial filling in or closing of the gap and rapid thermalization. As the system thermalizes in the correlated-band-insulating regime, it is interesting to compare the thermalization times from Figs. 2 and 3 with those observed in the metal (Fig. 1). 


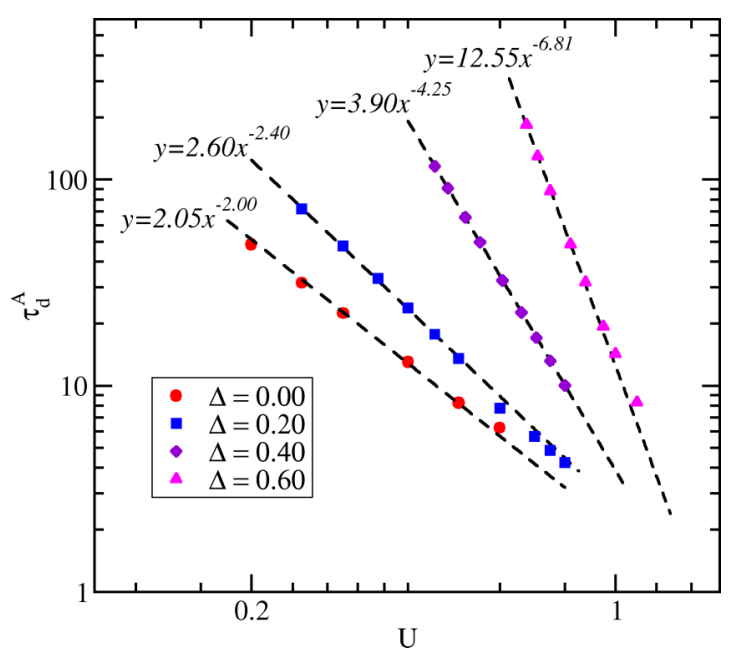

FIG. 6. Relaxation times of the double occupancy for different values of $\Delta$ and $U>\Delta$. The dashed curves indicate power-law fits.

In Fig. 6, we plot the thermalization times of the double occupancy for different values of the ionic potential and $U>$ $\Delta$. We find that the decrease of the thermalization time with $U$ in the correlated band insulator is faster compared than in the metal. The thermalization timescale is rather well described with the behavior $1 / U^{n}$ with $n>2(n=2$ for the metal), and the value of $n$ increases rapidly with the ionic potential. The large value of $n$ signifies that the thermalization dynamics of correlated band insulators is different from that of metals.

In Fig. 7 we show the spectrum $A^{R}(0)$ at the Fermi level as a function of $U / \Delta$ for different values of $\Delta$. For small $\Delta$, it becomes increasingly difficult to resolve the gap and density of states at the Fermi energy from a Fourier transform of real-time data because longer simulation times are needed. We have introduced an additional Gaussian kernel $\exp \left(-s^{2} \kappa\right)$ in the partial Fourier transform of the density of states in Eq. (10) to reduce the Fourier oscillations in a controlled way [32]. In

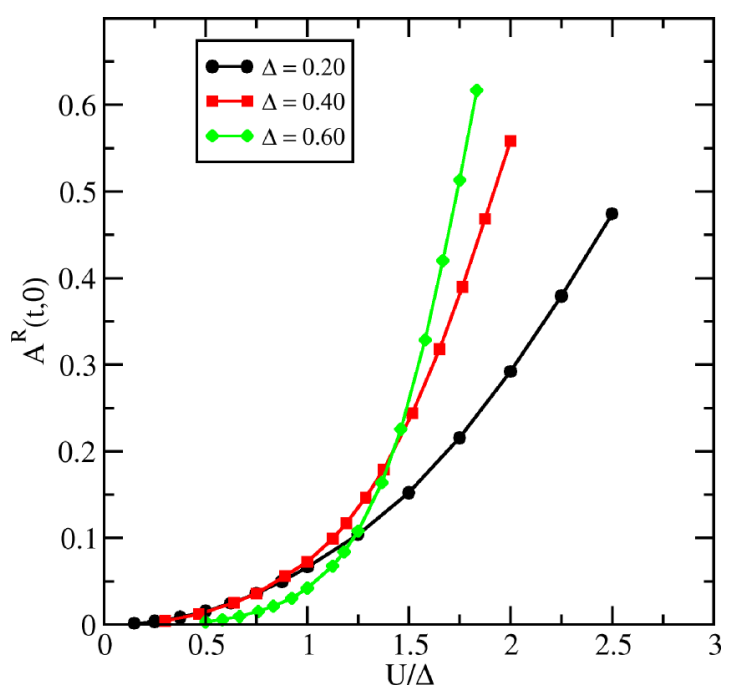

FIG. 7. The value of the retarded spectral function at $\omega=0$ for large time $t=180$ in the photoexcited state (excitation density such that $T_{\text {eff }}=1$ ).
Fig. 7, we choose $\kappa=0.0005$, and we have verified that the results shown do not depend on the cutoff within the size of the data symbol. A nonzero density of states at the Fermi level gives a finite phase-space volume for photodoped carriers to exchange the energy and thus can lead to fast thermalization of the photoexcited system. For $U \gtrsim \Delta$, the weight at the Fermi level increases strongly with $U / \Delta$. The larger exponent $n$ in Fig. 6 is therefore a consequence of the combined increase in the interaction $U$ and the spectral weight.

\section{B. Role of impact ionization}

Recently, it was shown that impact ionization processes can play an important role in the thermalization and relaxation of photoexcited Mott insulators [37]. Impact ionization occurs if the kinetic energy of charge carriers is larger than the size of the Mott gap, so that it is energetically allowed to create an additional doublon-hole pair via two-particle scattering. Impact ionization can be identified by the following characteristic signatures in the occupation function: (i) An impact ionization process decreases the occupied density of states at high energies and increases it at energies which are lower by at least the size of the gap. (ii) Impact ionization processes increase the density of mobile carriers and hence increase of integrated spectral weight above the gap.

To see whether impact ionization processes are important in the thermalization of correlated band insulators, we plot the time-dependent lesser spectral function for three different ratios of $U / \Delta=0.5,1.0$, and 1.375 (see Fig. 8). For $U / \Delta=$ 0.5 [Fig. 8(a)], the photoexcited system is not yet thermalized, and there is a well-defined gap in the retarded spectral function. In this regime the weight of the occupied density of states decreases at the lower edge of the conduction band, but the weight at the upper edge of the conduction band does not change at all, which is inconsistent with impact ionization. In the inset of Fig. 8(a), we show the total occupation above the Fermi level as a function of time. After photoexcitation, the integrated spectral weight decreases rapidly with time and reaches saturation at short timescales. We do not observe an increase at later times, which shows that impact ionization processes are not relevant on the timescale of our simulation when the local Coulomb interaction is smaller than the ionic potential.

In Fig. 8(b), we consider the case where the ionic potential is comparable to the Coulomb interaction $(U / \Delta=1.0)$. In this case, there is already some spectral weight at the Fermi level. One can observe that the occupied density of states increases at the lower band edge of the conduction band and decreases at the upper band edge, and the overall integrated spectral weight above $\omega=0$ increases at later times (insets in Fig. 8). Both signatures indicate impact ionization processes take place in this regime. Similarly, we can also identify signatures of the impact ionization process when $U$ is greater than the ionic potential, as shown in Fig. 8(c).

The precise time-dependent change in the occupied density of states can be used to quantify the significance of the impact ionization process at later times in the relaxation process. In Fig. 9, we show the difference in $A^{<}(t, \omega)$ between large times and some initial time $t=18$ for $U / \Delta=1.0$ and $t=24$ for $U / \Delta=1.375$. The time-dependent change in the occupied density of states is positive for frequencies below $\omega \sim 0.625$ 

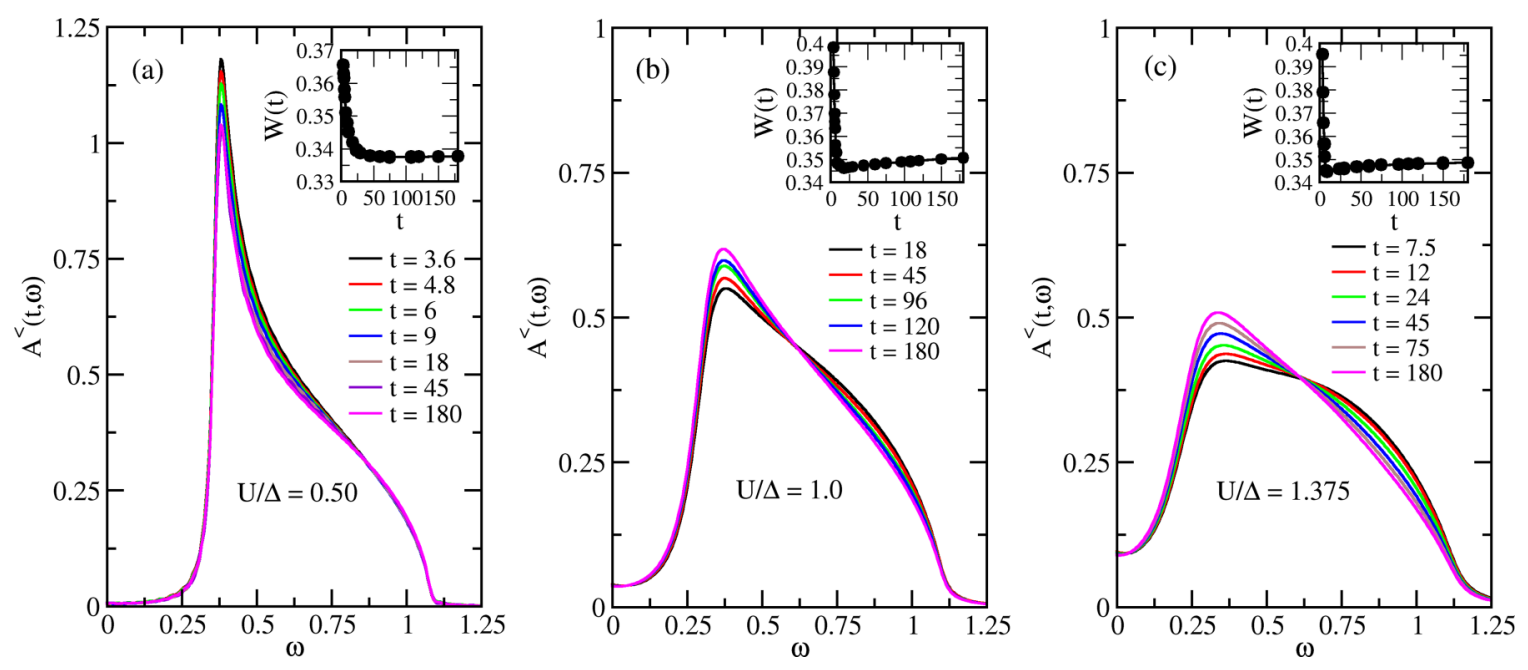

FIG. 8. Occupied density of states above the Fermi level at different times for $\Delta=0.4$ and $T_{\text {eff }}=1.0$. Insets: Integrated weight of the occupied density of states, $W(t)=\int_{0}^{\infty} A^{<}(t, \omega) d \omega$, above the Fermi level.

and negative above 0.625 . The ratio of the increase of the weight below $\omega=0.625$ to the decrease of weight above $\omega=0.625$ can therefore give an estimate of the number of mobile carriers produced in impact ionization processes. We find that this ratio is roughly 1.4 for $U / \Delta=1.0$ and 1.25 for $U / \Delta=1.375$, whereas relaxation only via impact ionization would suggest a ratio of 3 [37]. This implies that impact ionization processes are less significant in correlated band insulators than in small-gap Mott insulators. Probably, multiparticle-scattering mechanisms are also significant in the rapid thermalization of correlated band insulators.

\section{CONCLUSION}

In conclusion, we have studied the photoexcitation dynamics of correlated band insulators in the ionic Hubbard model.

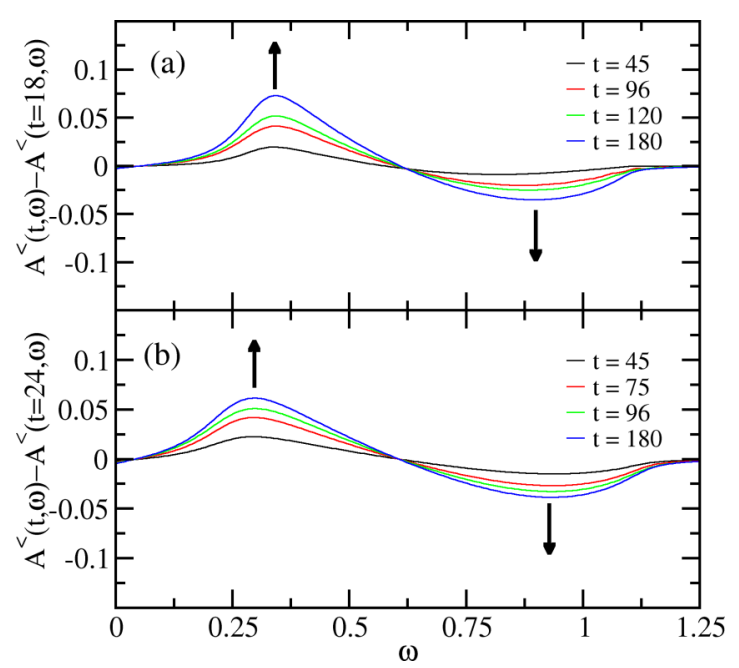

FIG. 9. Time-dependent change in the occupied density of states for excitation density corresponding to $T_{\text {eff }}=1.0, \Delta=0.4$, and (a) $U / \Delta=1.0$ or (b) $U / \Delta=1.375$. The up (down) arrows indicate frequencies at which a rapid increase (decrease) is observed more strongly during the relaxation.
Depending on the ratio of the interaction $U$ and the ionic potential $\Delta$, we observe a qualitatively different behavior: For $U \lesssim \Delta$, the spectrum itself is weakly influenced by the excitation (apart from a slight photoinduced screening of the gap, which is described by the Hartree shift of the bands). The relaxation of the system in this regime is characterized by intraband carrier scattering, leading to a nonthermal intermediate state with separate thermal distributions of electrons and holes. Above a crossover $U \approx \Delta$, the behavior changes. Photoexcitation can lead to a rapid renormalization of the spectrum, a filling in of the gap, and fast thermalization. This rapid thermalization of a small-gap insulator is reminiscent of the thermalization of small-gap Mott insulators [34], but in contrast to Mott insulators impact ionization processes [37] are less significant. The strong renormalization of the spectral function indicates that the dynamics of the correlated band insulator in this regime is no longer well described by mere quasiparticle scattering in rigid bands, which would be captured by kinetic equations.

In equilibrium, two systems with an identical gap in the spectral function can have very different ratios $U / \Delta$. For example, an insulator with $U / \Delta \gtrsim 1$ and a gap $\Delta_{*}$ can be compared to an ideal band insulator with ionic potential $\Delta_{*}$ and $U \ll \Delta_{*}$. The present analysis shows how these two systems with very similar equilibrium single-particle properties can be distinguished by their dynamical behavior. This may be used to classify weakly interacting insulators as either band insulators or correlated band insulators. In equilibrium, a qualitative distinction of correlated band insulators and band insulators may be based on different behaviors of spin and charge gaps $[9,10,22]$, and it will be interesting to see whether these pictures can be linked to the dynamical behavior. An important question is the role of spatial fluctuations for the thermalization dynamics, which are neglected in DMFT. For Mott insulators both short-range spin fluctuations [38] and charge fluctuations [36] can act as an energy bath for the electrons and thus affect the relaxation of distribution within the Hubbard band. Whether nonlocal fluctuations in the intermediate-coupling regime can also speed up interband thermalization and not 
only the intraband relaxation remains an important question for future investigations. The observed crossover from a nonthermal state to a thermal state can potentially be found in $\mathrm{SrRu}_{1-x} \mathrm{Ti}_{x} \mathrm{O}_{3}$, and some of the $3 d$ transition-metal oxides with crystal-field splitting [33].

[1] C. Giannetti, M. Capone, D. Fausti, M. Fabrizio, F. Parmigiani, and D. Mihailovic, Adv. Phys. 65, 58 (2016).

[2] D. Fausti, R. I. Tobey, N. Dean, S. Kaiser, A. Dienst, M. C. Hoffmann, S. Pyon, T. Takayama, H. Takagi, and A. Cavalleri, Science 331, 189 (2011).

[3] L. Stojchevska, I. Vaskivskyi, T. Mertelj, P. Kusar, D. Svetin, S. Brazovskii, and D. Mihailovic, Science 344, 177 (2014).

[4] I. Vaskivskyi, J. Gospodaric, S. Brazovskii, D. Svetin, P. Sutar, E. Goreshnik, I. A. Mihailovic, T. Mertelj, and D. Mihailovic, Sci. Adv. 1, e1500168 (2015).

[5] K. Nasu, Photoinduced Phase Transitions (World Scientific, Singapore, 2004).

[6] V. Tugushev, S. Caprara, and M. Avignon, Phys. Rev. B 54, 5466 (1996).

[7] S. Caprara, J. Phys.: Condens. Matter 10, 5389 (1998).

[8] S. Caprara, M. Avignon, and O. Navarro, Phys. Rev. B 61, 15667 (2000).

[9] A. P. Kampf, M. Sekania, G. I. Japaridze, and P. Brune, J. Phys.: Condens. Matter 15, 5895 (2003).

[10] G. I. Japaridze, R. Hayn, P. Lombardo, and E. Müller-Hartmann, Phys. Rev. B 75, 245122 (2007).

[11] S. R. Manmana, V. Meden, R. M. Noack, and K. Schönhammer, Phys. Rev. B 70, 155115 (2004).

[12] C. D. Batista and A. A. Aligia, Phys. Rev. Lett. 92, 246405 (2004).

[13] A. Garg, H. R. Krishnamurthy, and M. Randeria, Phys. Rev. Lett. 97, 046403 (2006).

[14] S. S. Kancharla and E. Dagotto, Phys. Rev. Lett. 98, 016402 (2007).

[15] L. Craco, P. Lombardo, R. Hayn, G. I. Japaridze, and E. MüllerHartmann, Phys. Rev. B 78, 075121 (2008).

[16] N. Paris, K. Bouadim, F. Hebert, G. G. Batrouni, and R. T. Scalettar, Phys. Rev. Lett. 98, 046403 (2007).

[17] K. Byczuk, M. Sekania, W. Hofstetter, and A. P. Kampf, Phys. Rev. B 79, 121103 (2009).

\section{ACKNOWLEDGMENTS}

We acknowledge fruitful discussions with Y. Mohammed and financial support from ERC Starting Grant No. 716648. The calculations were done at the PHYSnet cluster of the University of Hamburg.

[18] A. J. Kim, M. Y. Choi, and G. S. Jeon, Phys. Rev. B 89, 165117 (2014).

[19] A. Hoang, J. Phys.: Condens. Matter 22, 095602 (2010).

[20] A. Garg, H. R. Krishnamurthy, and M. Randeria, Phys. Rev. Lett. 112, 106406 (2014).

[21] P. Werner and A. J. Millis, Phys. Rev. Lett. 99, 126405 (2007).

[22] M. Sentef, J. Kuneš, P. Werner, and A. P. Kampf, Phys. Rev. B 80, 155116 (2009).

[23] A. Euverte, S. Chiesa, R. T. Scalettar, and G. G. Batrouni, Phys. Rev. B 87, 125141 (2013).

[24] G. Moeller, V. Dobrosavljević, and A. E. Ruckenstein, Phys. Rev. B 59, 6846 (1999).

[25] A. Fuhrmann, D. Heilmann, and H. Monien, Phys. Rev. B 73, 245118 (2006).

[26] S. S. Kancharla and S. Okamoto, Phys. Rev. B 75, 193103 (2007).

[27] M. Fabrizio, Phys. Rev. B 76, 165110 (2007).

[28] H. Hafermann, M. I. Katsnelson, and A. I. Lichtenstein, Europhys. Lett. 85, 37006 (2009).

[29] H. Haug and A. P. Jauho, Quantum Kinetics in Transport and Optics of Semiconductors, Springer Series in Solid-State Sciences Vol. 123 (Springer, Berlin, 2008).

[30] M. Wais, M. Eckstein, R. Fischer, P. Werner, M. Battiato, and K. Held, arXiv:1806.02570.

[31] H. Aoki, N. Tsuji, M. Eckstein, M. Kollar, T. Oka, and P. Werner, Rev. Mod. Phys. 86, 779 (2014).

[32] M. Eckstein, M. Kollar, and P. Werner, Phys. Rev. B 81, 115131 (2010).

[33] K. Maiti, R. S. Singh, and V. R. R. Medicherla, Phys. Rev. B 76, 165128 (2007).

[34] M. Eckstein and P. Werner, Phys. Rev. B 84, 035122 (2011).

[35] J. K. Freericks, H. R. Krishnamurthy, and T. Pruschke, Phys. Rev. Lett. 102, 136401 (2009).

[36] D. Golež, L. Boehnke, H. U. R. Strand, M. Eckstein, and P. Werner, Phys. Rev. Lett. 118, 246402 (2017).

[37] P. Werner, K. Held, and M. Eckstein, Phys. Rev. B 90, 235102 (2014).

[38] M. Eckstein and P. Werner, Sci. Rep. 6, 177 (2016). 